\title{
THERMAL STRESS WAVE AND SPALLATION INDUCED BY AN ELECTRON BEAM
}

\author{
Y.C. ZHOU ${ }^{\dagger} \ddagger$ Z.P. DUAN ${ }^{\dagger}$ and X. H. YAN ${ }^{\ddagger}$ \\ ${ }^{\dagger}$ Laboratory for Laser and Dynamic Behaviour of Materials Institute of Mechanics, CAS, Beijing, 100080, \\ People's Republic of China \\ ${ }^{\ddagger}$ Department of Physics, Xiangtan University, 411105 , Xiangtan Hunan, People’s Republic of China
}

(Received 21 September 1995; in revised form 11 October 1996)

\begin{abstract}
Summary-Thermal stress wave and spallation in aluminium alloy exposed to a high fluency and low energy electron beams are studied theoretically. A simple model for the study of energy deposition of electrons in materials is presented on the basis of some empirical formulae. Under the stress wave induced by energy deposition, microcracks and/or microvoids may appear in target materials, and in this case, the inelastic volume deformation should not vanish. The viscoplastic model proposed by Bodner and Partom with corresponding Gurson's yield function requires modification for this situation. The new constitutive model contains a scalar field variable description of the material damage which is taken as the void volume fraction of the polycrystalline material. Incorporation of the damage parameter permits description of rate-dependent, compressible, inelastic deformation and ductile fracture. The melting phenomenon has been observed in the experiment, therefore one needs to take into account the melting process in the intermediate energy deposition range. A three-phase equation of state used in the paper provides a more detailed and thermodynamical description of metals, particularly, in the melting region. The computational results based on the suggested model are compared with the experimental test for aluminium alloy, which is subjected to a pulsed electron beam with high fluency and low energy. (C) 1997 Elsevier Science Ltd.
\end{abstract}

Keywords: thermal stress wave, spallation, electron beam, energy deposition, constitutive model.

\section{INTRODUCTION}

In the fields of radiation hardening and nuclear electricity generating plants, it is useful to study thermal mechanical effects due to rapid energy deposition, including that of $\mathrm{X}$ ray energy from nuclear explosions and electron beam machines, or exploding wire, magnetic flux compression etc. All of the phenomena characterized by high energy densities may take place within a few nanoseconds to a hundred nanoseconds. In the recent studies [1-5] the focus has been given on the dynamic response of materials to the intense thermal stress generated by powerful pulsed lasers or electron beams. The phenomena of the melting, vaporization as well as the plasma generation on the surface of the target material in the incident particles-materials interaction are different from that in the mechanical loading. Although dynamic loading conditions are different, shock wave phenomena produced by dynamic loadings are commonly the same in both cases. This strong shock wave may lead the spallation due to the wave reflection from the rear free surface of the targets. To model the thermal shock wave and spall damage one must consider the interaction of incident electrons with materials, the surface melting, the propagating of stress waves as well as the dynamic damage and fracture in solids.

The energy deposition function in the case of electron beam lithography [6], electron beam weld penetration [7] is a fundamental problem. Generally, the Monte-Carlo method has been widely used to calculate the pulsed energy deposition process of incident electron beam [8]. In recent years, Glezos and co-workers [6,9] developed an analytical model for the calculation of the energy deposition function in the case of an electron point beam incident at a right angle over a composite substrate. The model uses the diffusion approximation to the Boltzmann transport equation as a starting point for a self-consistent calculation. To avoid the complicated numerical calculation we present first a simple model for the energy deposition of pulsed electron beams in materials. The melting process is important for the study of material dynamic response in the intermediate energy deposition range. The Mie-Grüneisen equation of state should not be applied to the intermediate range of pressure and temperature [10]. A more complicated three-phase equation of state has been developed by Royce [11] for the treatment of metals in the intermediate range. The three-phase model offers an advantage of explicit treatment of melting transition sophisticatedly. 
The Bodner-Partom model introduces the microvoid volume fraction to describe material degradation $[12,13]$. The void volume fraction can be directly incorporated into the constitutive equations, and serves as a damage variable to quantify the degradation processes of ductile materials. The Bonder-Partom model only holds that the voided material is plastically incompressible, however, this is not true due to the presence of voids. This paper would make further attempt to modify the Bodner-Partom constitutive theory [12-15] by introducing Gurson's flow potential [16].

The processes of dynamic damage in aluminium alloy exposed to a high fluency and low energy pulsed electron beam are successfully simulated with a finite-difference dynamic code. Following this introductory section, the simple model on energy deposition of electrons in target materials is presented. A modified Bonder-Pardom's constitutive model including damage is discussed in detail in Section 3. One-dimensional finite-difference method and related results are given in Section 4 and Section 5, respectively; Section 6 gives our conclusions.

\section{ENERGY DEPOSITION}

When a pulsed electron beam radiates on a target, one or some of collision processes described below may take place, if the nuclear reactions and other strong interactions are not taken into account:

1. inelastic collisions with atomic electrons;

2. inelastic collisions with nuclei;

3. elastic collisions with atomic electrons;

4. elastic collisions with nuclei.

The energy diminution and deflection on the incident particles' motion could be brought about in these four courses.

Inelastic collisions are the primary mechanism governing the beta particles (incident electrons) losing energy in matter. Usually, the inelastic collisions with atomic electrons result in excitation or freeing of the atomic electrons. This first kind of collision is sometimes called ionization. Inelastic collisions with nuclei, on the other hand, result in the deflections of the incident beta particles. During the deflection, a portion of electromagnetic radiation (bremsstrahlung) may be emitted. The kinetic energy of the beta particle is reduced by an amount equal to the bremsstrahlung energy. The energy losses due to the second kind of collision are sometimes called to be radiative. The first kind of loss plays a dominating role at low beta energy, and contrarily, the second kind of radiative loss dominating at high beta energy.

In elastic collisions with electrons, the incident beta particles are deflected but do not lose their energy. During the elastic collision with a nucleus, the beta particles only lose their kinetic energy but keep the momentum conserved between the colliding particles. In an elastic collision between the incident and atomic electrons, the total energy and momentum are conserved, and in general, not enough energy could be transferred to excite the struck electron to become fully free.

The colliding process occurs usually as a stochastic event. The probability of each type of encounter can be calculated from scattering theory. Many investigators made use of the Monte-Carlo method to study the interaction of an electron beam with matter. Sometimes, the method seems to be quite complicated in numerical simulation. We present a simple model on the basis of the empirical formulae for both the effective mean range as suggested by Rudie [8] and the transmissive coefficient discussed by Subba [17].

Consider an electron beam impinges on a target with $N_{0}(t)$ electrons per unit area and per unit time. The incident electron beam is multienergetic and is assumed to be consist of $k$ kinds of energy. $\varepsilon_{0 j}$ is the fraction of the energy $E_{0 j}(j=1,2,3, \ldots, k)$. The penetration depth in the incident direction is defined as the effective mean range $R_{0 j}$. For the low energy electron beam and the target with low atomic number $Z$, the following empirical formula for the effective mean range $R_{0 j}$ (in $\mathrm{g} / \mathrm{cm}^{2}$ )

$$
R_{0 j}=G_{1} E_{0 j}^{G_{2}-G_{3} \ln E_{0 j}}
$$

is a function of kinetic energy $E_{0 j}$ (in $\mathrm{MeV}$ ) was proposed by Rudie [8]. The penetration depth from the incident surface $x_{3}(\mathrm{~cm})$ in the incident direction is defined as remained effective mean range $R_{j}\left(\right.$ in $\left.\mathrm{g} / \mathrm{cm}^{2}\right)$ :

$$
R_{j}=G_{1} E_{j}^{G_{2}-G_{3} \ln E_{j}}
$$


where $E_{j}$ is kinetic energy and $G_{1}, G_{2}$ and $G_{3}$ are material constants and $G_{1}=0.5493, G_{2}=1.216$, $G_{3}=0.11$ for aluminium [8].

From Eqns (1) and (2), the kinetic energy $E_{j}$ at the distance $x_{3}$ away from the surface $x_{3}=0$ is expressed by

$$
E_{j}=\exp \left\{\frac{0.5}{G_{3}}\left[G_{2}-\left(G_{2}^{2}-4 G_{3} \ln \frac{R_{0 j}-\rho x_{3}}{G_{1}}\right)^{1 / 2}\right]\right\} .
$$

On the basis of many theoretical and experimental data Subba [17] presented an empirical expression for the transmissive coefficient in the incident direction of the electrons as follows:

$$
T_{j}=\frac{1+\exp \left(-\kappa \kappa_{0}\right)}{1+\exp \left[\kappa\left(\rho x_{3}-\kappa_{0} R_{0 j}\right) / R_{0 j}\right]},
$$

where $\kappa=9.2 Z^{-0.2}+16 Z^{-2.2}, \kappa_{0}=0.63(Z / A)+0.27, Z$ is the atomic number, $A$ the atomic weight (in $\mathrm{g} / \mathrm{mole}$ ) and $\rho$ the material density.

When the dynamic coupling effect of energy deposition and the beam induced stress wave is considered, the deposited specific energy rate $E_{\mathrm{D}}$ (in $\mathrm{MeV} / \mathrm{g} \mathrm{s}$ ) can be calculated from Eqns (3) and (4) through

$$
E_{\mathrm{D}}=-\sum_{j=1}^{k} \varepsilon_{0 j} N_{0}(t) \frac{\partial}{\partial x_{3}}\left(\frac{T_{j} E_{j}}{\rho}\right),
$$

which will play an important role in the study of the beam induced stress wave propagation, as issued in the Sections 4 and 5. The readers who are not familiar with the approximate expressions used for the deposited energy from an electron beam are referred to reference [8].

\section{CONSTITUTIVE MODEL}

Material degradation due to the increase in microstructural deterioration is irreversible and history dependent. The theory of solid thermodynamics for irreversible processes gives a useful framework for building an appropriate constitutive model. The internal variable method is usually introduced to characterize the history dependence $[18,19]$. The following discussion will focus mainly on the viscoplastic material and its mechanical behaviour with isotropic damage. The damage variable is denoted by the void fraction $\xi=1-V_{s} / V=1-\rho / \rho_{s}$, where $V$ and $\rho$ are, respectively, the macroscopic specific volume and density for the voided materials, $V_{s}$ and $\rho_{s}$ are the corresponding quantities for the material without voids. Because of the presence of microvoids, the elastic modulus are assumed to be given by [20]:

$$
\begin{gathered}
\bar{\mu}=\mu(1-\xi)\left(1-\frac{6 K+12 \mu}{9 K+8 \mu} \xi\right), \\
\bar{\nu}=\frac{1}{2} \frac{3 \bar{K}-2 \bar{\mu}}{3 \bar{K}+\bar{\mu}}, \quad \bar{K}=\frac{4 \mu K(1-\xi)}{4 \mu+3 K \xi},
\end{gathered}
$$

where $\bar{\mu}$ is the shear moduli, $\bar{\nu}$ the Poisson's ratio and $\bar{K}$ the elastic bulk and correspondingly, the elastic bulk $K$, shear moduli $\mu$ and Poisson's ratio $\nu$ are denoted for the solid without voids.

The rate of deformation tensor $\mathbf{D}$ defined as the symmetric part of $\dot{\mathbf{F}} \cdot \mathbf{F}^{-1}$, where $\mathbf{F}$ is the deformation gradient and can be decomposed into an elastic part $\mathbf{D}^{\mathbf{e}}$ and an inelastic part $\mathbf{D}^{\text {in }}$,

$$
\mathbf{D}=\mathbf{D}^{\mathbf{e}}+\mathbf{D}^{\text {in }} .
$$

The rate of elastic deformation for the voided solid is given by:

$$
\mathbf{D}^{\mathbf{e}}=\frac{1}{2 \bar{\mu}}\left[\hat{\boldsymbol{\sigma}}-\frac{\bar{\nu}}{1+\bar{\nu}}(\hat{\boldsymbol{\sigma}}: \mathbf{I}) \mathbf{I}\right],
$$

where $\mathbf{I}$ is the second-order identity tensor and the notation $\mathbf{A}: \mathbf{B}$ denotes the dyadic product between tensor $\mathbf{A}$ and $\mathbf{B}$. In Eqn (8), $\mathbf{D}^{\mathfrak{e}}$ is a linear function of the objective stress rate of the Cauchy tensor $\hat{\boldsymbol{\sigma}}$, which is expressed by

$$
\hat{\boldsymbol{\sigma}}=\dot{\boldsymbol{\sigma}}+\boldsymbol{\sigma} \cdot \mathbf{W}-\mathbf{W} \cdot \boldsymbol{\sigma},
$$


where the dot over a character signifies the material time derivative:

$$
\dot{\sigma}=\frac{\partial \boldsymbol{\sigma}}{\partial \mathrm{t}}+\mathbf{v} \cdot(\operatorname{grad} \sigma)
$$

and $\mathbf{W}$ is considered as a generalized spin tensor and $\mathbf{v}$ the particle velocity.

The plastic response for the voided material is modelled using a plastic yield function as proposed by Gurson [16]. With the void volume fraction $\xi$ and the average strength of the matrix material $\bar{\sigma}_{\mathrm{F}}$, the plastic flow potential is written as

$$
\Phi=\frac{1}{2} \mathbf{S}: \mathbf{S}+\frac{2}{3} \sigma_{F}^{2} q_{1} f^{*} \cosh \left(\frac{q_{2} \boldsymbol{\sigma}: \mathbf{I}}{2 \sigma_{\mathrm{F}}}\right)-\frac{1}{3} \sigma_{\mathrm{F}}^{2}\left(1+q_{1}^{2} f^{* 2}\right)=0,
$$

where the deviator stress $\mathbf{S}$ and deviator stress invariant $J_{2}$ are, respectively, expressed by

$$
\mathbf{S}=\boldsymbol{\sigma}-\frac{1}{3}(\boldsymbol{\sigma}: \mathbf{I}) \mathbf{I}=\boldsymbol{\sigma}+P \mathbf{I}, \quad J_{2}=\frac{1}{2} \mathbf{S}: \mathbf{S} .
$$

The parameters $q_{1}$ and $q_{2}$ in Eqn (12) were introduced by Tvergaard [21,22] who attempted to bring the model into closer agreement with full numerical analyses in the case of periodic arrays of voids. The function $f^{*}$ accounts for the effects of rapid void coalescence and takes to the form [23]:

$$
f^{*}= \begin{cases}\xi & \xi \leq f_{c} \\ f_{c}+\left[\left(f_{\nu}^{*}-f_{c}\right) /\left(f_{\mathrm{F}}-f_{c}\right)\right]\left(\xi-f_{c}\right) & \xi>f_{c}\end{cases}
$$

where $f_{\nu}^{*}=1 / q_{1}$ and the parameters $q_{1}=1.5, q_{2}=1.0, f_{c}=0.15$ and $f_{\mathrm{F}}=0.25$ for aluminium alloys.

The increases of the void volume fraction $\xi$ from the initial value $\xi_{0}$ are characterized by several physical mechanisms such as void diffusion, void nucleation and void growth. The contribution of void diffusion becomes significant, however, only at elevated temperatures. Therefore, the rate of accumulation of microdamage at nonelevated temperatures can be essentially represented through the void nucleation and growth

$$
\dot{\xi}=\dot{\xi}_{\text {growth }}+\dot{\xi}_{\text {nucleation }} \text {. }
$$

The growth of existing voids with deformation is determined from the condition of plastic incompressibility of the matrix material:

$$
\dot{\xi}_{\text {growth }}=(1-\xi) \mathbf{D}^{\text {in }}: \mathbf{I} \text {. }
$$

The nucleation law fully controlled by plastic strain is simply modeled as

$$
\dot{\xi}_{\text {nucleation }}=\psi \cdot\left(\dot{\sigma}_{\mathrm{F}}+\frac{1}{3} \dot{\boldsymbol{\sigma}}: \mathbf{I}\right)
$$

when $\left[\dot{\sigma}_{\mathrm{F}}+(1 / 3 \dot{\sigma}: \mathbf{I}]\right.$ is positive, otherwise $\dot{\xi}_{\text {nucleation }}=0$. The parameter $\psi$ in Eqn (17) is a function of $\left[\sigma_{\mathrm{F}}+(1 / 3) \sigma: \mathbf{I}\right]$. Following Chu and Needleman [24], $\psi$ is specified as a normal distribution in terms of mean nucleation stress such that

$$
\psi=\frac{f_{\mathrm{N}}}{\sigma_{0} s_{\mathrm{N}} \sqrt{2 \pi}} \exp \left[-\frac{1}{2}\left(\frac{\left(\sigma_{\mathrm{F}}+\frac{1}{3} \boldsymbol{\sigma}: \mathbf{I}\right)-\sigma_{\mathrm{N}}}{\sigma_{0} s_{\mathrm{N}}}\right)^{2}\right],
$$

where $\sigma_{0}$ is the reference stress, $f_{\mathrm{N}}$ the volume fraction of void nucleating particles, $\sigma_{\mathrm{N}}$ the mean nucleation stress, and $s_{N}$ the standard deviation from the normal distribution.

For visco-plastic materials, a plastic strain rate always depends on the current state regardless of the direction of the stress rate. Gurson's yield function $\Phi$ could now serve as the plastic potential function, of which the partial differentials in stress space give the inelastic strain rate tensor as

$$
\mathbf{D}^{\mathrm{in}}=\Lambda \frac{\partial \Phi}{\partial \boldsymbol{\sigma}}
$$

where $\Lambda$ is a proportionality factor. Squaring Eqn (19) leads to

$$
D_{2}^{\text {in }}=\frac{1}{2} \Lambda^{2} \frac{\partial \Phi}{\partial \sigma}: \frac{\partial \Phi}{\partial \sigma}=\Lambda^{2} J_{2}^{\prime}
$$

where $D_{2}^{\text {in }}$ and $J_{2}^{\prime}$ are, respectively, the inelastic strain rate and deviatoric stress-like invariants and are 
given by

$$
D_{2}^{\text {in }}=\frac{1}{2} \mathbf{D}^{\text {in }}: \mathbf{D}^{\text {in }}, \quad J_{2}^{\prime}=\frac{1}{2} \frac{\partial \Phi}{\partial \boldsymbol{\sigma}}: \frac{\partial \Phi}{\partial \boldsymbol{\sigma}} .
$$

The relation governing inelastic deformation is called the "kinetic equation" $D_{2}^{\text {in }}=F\left(J_{2}^{\prime}\right)$, and its special form for voided material originally proposed by Bodner [12,13] based on the Bodner-Partom model $[14,15]$ can be modified as

$$
D_{2}^{\mathrm{in}}=D_{0}^{2} \exp \left\{-\left[B^{2}(1-\xi)^{2} / 3 J_{2}^{\prime}\right]^{n}\right\}
$$

where $J_{2}$ in Bodner's original formula $[12,13]$ has been replaced by $J_{2}^{\prime}$ in order to assure that the plastic volume rate is compressible; $D_{0}^{2}$ is the limiting strain rate in shear; $n$ a material constant; and $B$ is interpreted as a load history dependent parameter that represents the hardened state of the material variable.

$$
\begin{gathered}
\dot{B}=m\left(B_{1}-B\right) \dot{W}_{p}, \\
\dot{W}_{p}=\boldsymbol{\sigma}: \mathbf{D}^{\text {in }}, \quad B(0)=B_{0}, \quad W_{p}(0)=0 .
\end{gathered}
$$

Combining Eqns (19)-(22), for the viscoplastic materials considered here, the macroscopic plastic strain rate is determined by the present stress and deformation state from the following equation:

$$
\mathbf{D}^{\text {in }}=\left\{\frac{D_{0}^{2}}{J_{2}^{\prime}} \exp \left\{-\left[B^{2}(1-\xi)^{2} / 3 J_{2}^{\prime}\right]^{n}\right\}\right\}^{1 / 2} \frac{\partial \Phi}{\partial \boldsymbol{\sigma}} .
$$

As a consequence, we could see again that the stress term is no longer deviatoric so that the plastic strain rate could have a compressible component. However, in the original Bodner-Partom treatment of damage [12,13], the flow law remained unchanged from the Prandtl-Reuss form so that it is obvious that plastic incompressibility was retained. This is also a reason for our modification from Bodner-Partom original formula to Eqn (22). From the flow potential expression (12), we have

$$
\begin{gathered}
\frac{\partial \Phi}{\partial \boldsymbol{\sigma}}=\mathbf{S}+\frac{1}{3} q_{1} q_{2} \sigma_{F} f^{*} \sinh \left(\frac{q_{2} \boldsymbol{\sigma}: \mathbf{I}}{2 \sigma_{F}}\right) \mathbf{I}, \\
J_{2}^{\prime}=\frac{1}{2} \mathbf{S}: \mathbf{S}+\frac{1}{6}\left[q_{1} q_{2} \sigma_{F} f^{*} \sinh \left(\frac{q_{2} \boldsymbol{\sigma}: \mathbf{I}}{2 \sigma_{F}}\right)\right]^{2} .
\end{gathered}
$$

Also, from Eqn (12), the evolution rate for $\sigma_{F}$, the equivalent flow strength of the matrix is given as

$$
\dot{\sigma}_{F}=-\left(\frac{\partial \Phi}{\partial \boldsymbol{\sigma}}: \hat{\boldsymbol{\sigma}}+\frac{\partial \Phi}{\partial \xi}\right) / \frac{\partial \Phi}{\partial \sigma_{F}}
$$

where we calculate

$$
\begin{gathered}
\frac{\partial \Phi}{\partial \xi}=\frac{2}{3} \sigma_{F}^{2} q_{1} \frac{\partial f^{*}}{\partial \xi} \cosh \left(\frac{q_{2} \boldsymbol{\sigma}: \mathbf{I}}{2 \sigma_{F}}\right)-\frac{2}{3} \sigma_{F}^{2} q_{1}^{2} \frac{\partial f^{*}}{\partial \xi} f^{*} \\
\frac{\partial \Phi}{\partial \sigma_{F}}=\frac{4}{3} q_{1} \sigma_{F} f^{*} \cosh \left(\frac{q_{2} \boldsymbol{\sigma}: \mathbf{I}}{2 \sigma_{F}}\right)-\frac{1}{3} q_{1} q_{2} f^{*} \sinh \left(\frac{q_{2} \boldsymbol{\sigma}: \mathbf{I}}{2 \sigma_{F}}\right) \boldsymbol{\sigma}: \mathbf{I}-\frac{2}{3} \sigma_{F}\left(1+q_{1}^{2} f^{* 2}\right) .
\end{gathered}
$$

From Eqns (25)-(27), the plastic volume deformation rate takes the form

$$
\mathbf{D}^{\text {in }}: \mathbf{I}=\left\{\frac{D_{0}^{2}}{J_{2}^{\prime}} \exp \left\{-\left[B^{2}(1-\xi)^{2} / 3 J_{2}^{\prime}\right]^{n}\right\}\right\}^{1 / 2} q_{1} q_{2} \sigma_{F} f^{*} \sinh \left(\frac{q_{2} \boldsymbol{\sigma}: \mathbf{I}}{2 \sigma_{F}}\right) .
$$

Eqn (31) signifies once again that the plastic volume deformation rate for the voided material does not vanish as long as the void volume fraction, or damage variable $\xi$ does exist.

\section{ONE-DIMENSIONAL FINITE-DIFFERENCE CALCULATION}

The motion of points of the target material induced by an electron beam radiation is assumed to be one-dimensional:

$$
x_{1}=X_{1}, x_{2}=X_{2}, x_{3}=f\left(X_{3}, t\right),
$$


where $x_{1}, x_{2}$, and $x_{3}$ are the Eulerian coordinates, $X_{1}, X_{2}$, and $X_{3}$ represent their corresponding Lagrangian coordinates and $t$ is time. The function $f\left(X_{3}, t\right)$ is assumed to be positive, continuous and differentiable. The particle velocity, rate of deformation and stress tensors have only the following nontrival components:

$$
\nu_{3} \neq 0, \quad D_{33}=\frac{\partial v_{3}}{\partial x_{3}}, \quad \sigma_{11}=\sigma_{22} \neq 0, \quad \sigma_{33} \neq 0,
$$

and the spin tensors $\mathbf{W}=0$. The one-dimensional flow governing equations are:

$$
\begin{array}{cl}
\text { Continuity : } & \frac{\dot{V}}{V}=\frac{\partial v_{3}}{\partial x_{3}}, \\
\text { Momentum : } & \frac{\partial \sigma_{33}}{\partial x_{3}}=\frac{\dot{v}_{3}}{V}, \\
\text { Energy : } \quad \dot{e}=\sigma_{33} \dot{V}+E_{D}\left(x_{3}, t\right),
\end{array}
$$

where $e$ is specific energy. Eqns (25)-(28) give together the following elastic-viscoplastic damage constitutive rate equations:

$$
\begin{gathered}
-\frac{1}{4 \bar{\mu}}\left[\dot{\sigma}_{33}+\frac{3(1-\bar{\nu})}{1+\bar{\nu}} \dot{P}\right]+\left(\frac{D_{0}^{2}}{J_{2}^{\prime}} \exp \left\{-\left[B^{2}(1-\xi)^{2} / 3 J_{2}^{\prime}\right]^{n}\right\}\right)^{1 / 2} \\
\times\left[-\frac{1}{2}\left(P+\sigma_{33}\right)+\frac{1}{3} q_{1} q_{2} \sigma_{F} f^{*} \sinh \left(-\frac{3 q_{1} P}{2 \sigma_{F}}\right)\right]=0 \\
\frac{1}{2 \bar{\mu}}\left(\sigma_{33}+\frac{3 \bar{\nu}}{(1+\bar{\nu})} \dot{P}\right)+\left(\frac{D_{0}^{2}}{J_{2}^{\prime}} \exp \left\{-\left[B^{2}(1-\xi)^{2} / 3 J_{2}^{\prime}\right]^{n}\right\}\right)^{1 / 2} \\
\times\left[P+\sigma_{33}+\frac{1}{3} q_{1} q_{2} \sigma_{F} f^{*} \sinh \left(-\frac{3 q_{2} P}{2 \sigma_{F}}\right)\right]=\frac{\partial \nu_{3}}{\partial x_{3}} \\
\dot{\sigma}_{F}=-\left\{\frac{3}{2}\left(P+\sigma_{33}\right)\left(\dot{P}+\dot{\sigma}_{33}\right)-q_{1} q_{2} \sigma_{F} f^{*} \dot{P} \sinh \left(-\frac{3 q_{2} P}{2 \sigma_{F}}\right)\right. \\
\left.+\frac{2}{3} \sigma_{F}^{2} q_{1} \frac{\partial f^{*}}{\partial \xi}\left[\cosh \left(-\frac{3 q_{2} P}{2 \sigma_{F}}\right)-q_{1} f^{*}\right] \dot{\xi}\right\} \\
\times\left[\frac{4}{3} \sigma_{F} q_{1} f^{*} \cosh \left(-\frac{3 q_{2} P}{2 \sigma_{F}}\right)+q_{1} q_{2} P f^{*} \sinh \left(-\frac{3 q_{2} P}{2 \sigma_{F}}\right)-\frac{2}{3} \sigma_{F}\left(1+q_{1}^{2} f^{* 2}\right)\right]^{-1}, \\
J_{2}^{\prime}=\frac{3}{4}\left(P+\sigma_{33}\right)^{2}+\frac{1}{6}\left[q_{1} q_{2} \sigma_{F} f^{*} \sinh \left(-\frac{3 q_{2} P}{2 \sigma_{F}}\right)\right]^{2}
\end{gathered}
$$

It is assumed that the equation of state relating pressure $P$, specific volume $V$ and specific internal energy $e$ for the matrix in voided material possesses the same form as in nonvoided material $[25,26]$. With this assumption, the equation of state gives the pressure $P$ as a function of specific volume $V$, specific internal energy $e$, and porosity $\xi$. In general, the material subjected to a pulsed electron beam is in the intermediate state, i.e. pressures and temperatures near the melting transition. Melting must be adequately accounted for in material response calculations in the intermediate energy deposition range. The three-phase equation of state [11] offers an advantage of explicit treatment of melting transition sophisticatedly, which takes the form without damage

$$
P=F(V, e) \text {. }
$$

Correspondingly, for the damage material, we have

$$
P=\alpha P_{s}=\alpha F(\alpha V, e),
$$

where $\alpha=1-\xi$.

Micrographic examination for the spalled target sample exhibit a ductile type of fracture, and the failure mode shows the final stage of the void growth process $[20,27,31]$. This suggested that a local criterion for ductile spallation can be defined using a critical value for the void volume fraction, $\xi=\xi_{F}$, at which the material element (point) experiences separation. In other words, the material relinquishes its 
Table 1. Material constants for aluminium alloy

$\rho_{0}=2.78 \mathrm{~g} / \mathrm{cm}^{2}[11]$

$K=62.0 \mathrm{GPa}[20]$

$B_{1}=0.869 \mathrm{GPa}[30]$

$\sigma_{0}=0.245 \mathrm{GPa}[24,28]$

$f_{N}=0.04[24,28]$

$\left(D_{0}^{2}\right)^{1 / 2}=10^{4} \mathrm{~s}^{-1}[30]$

$q_{1}=1.5[23]$

$n=5.0[30]$

$\xi_{F}=0.25[20,27]$

$A=27.74[29]$

$G_{2}=1.216[8]$

$$
\begin{aligned}
& \mu=28.6 \mathrm{GPa}[20] \\
& B_{0}=0.682 \mathrm{GPa}[30] \\
& m=87.0(\mathrm{GPa})^{-1}[30] \\
& \sigma_{N}=0.3 \mathrm{GPa}[24,28] \\
& s_{N}=0.06[24,28] \\
& \nu=0.3[20] \\
& q_{2}=1.0[23] \\
& \xi_{0}=10^{-3}[20] \\
& Z=13.72[29] \\
& G_{1}=0.5493[8] \\
& G_{3}=0.11[8]
\end{aligned}
$$

ability to maintain stress. Application of the elastic-viscoplastic-damage constitutive equations needs the material parameters to be known. Table 1 provides a summary of the material constants involved in the constitutive model. The three-phase equation of state used in our calculation for the aluminium alloy can also be found in reference [11].

\section{RESULTS AND DISCUSSION}

In this section results concerning the stress wave propagation are given as detailed as possible. A comparison for the aluminium alloy is also made of the experimental data with numerical results.

First, the experiments were carried out with a $1 \mathrm{MV}$ electron beam machine [32]. The experimental condition is listed below: The voltage in diode is $0.67 \mathrm{MV}$ and current intensity is $16 \mathrm{KA}$. The cathodeto-anode gap in the field emission tubes is $12 \mathrm{~mm}$ and the vacuum is $6.7 \times 10^{-3} \mathrm{~Pa}$. This operation mode could produce a beam with an approximately constant energy distribution and the mean energy of the electrons is $0.267 \mathrm{Mev}$. This beam is multienergetic with energy spectrum illustrated in Fig. 1. The fluency of electron beam is $32 \mathrm{cal} / \mathrm{cm}^{2}$ and the full width at half-maximum is $25 \mathrm{~ns}$.

The temporal shape of emission electric current $I(t)$ in diode is similar to sinusoidal form as confirmed from the measurements. Therefore, the pulsed electron beam when hitting upon the target plate owns presumably a same temporal shape as the emission current in diode. We take $I(t)=I_{0} \sin (\omega t)$ with the peak current intensity $I_{0}=16 \mathrm{KA}, \omega=2 \pi / 75 \mathrm{~ns}^{-1}$ and the pulse duration $37.5 \mathrm{~ns}$.

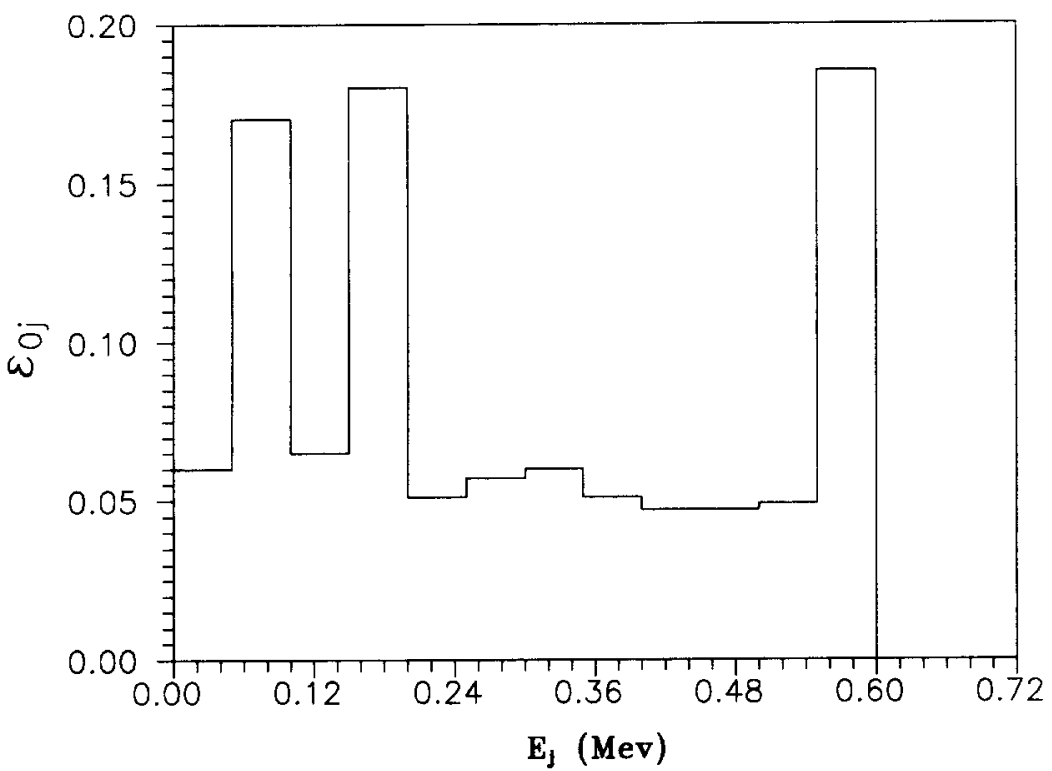

Fig. 1. Energy spectrum of an electron beam. 
The target samples, i.e. the anode, are the disks of aluminium alloy $30 \mathrm{~mm}$ in diameter. The beam spot is $10 \mathrm{~mm}$ in diameter. A quartz crystal is closely struck on the rear surface to measure the normal velocity histories of the surface. The disks with five different thicknesses, $1.2,1.5,2.0,2.8$, and $4.0 \mathrm{~mm}$, are used in order to observe the amplitude of stress wave in different shot and to compare them with theoretical predictions.

When a monoenergetic electron beam with $1 \mathrm{Mev}$ energy impinges normally on aluminium target, the specific energy $Q\left(\mathrm{Mev} / \mathrm{g} \mathrm{cm}^{2}\right)$ deposited on per unit area is illustrated in Fig. 2. To check the simple model of energy deposition Eqn (5), we also compared the calculated results of the energy deposition with the experimental data given in reference [8]. As seen in Fig. 2, the magnitude of the calculated energy deposition agree approximately with the measured values.

The specific energy taken at the time of ending of electron beam radiation is illustrated in Fig. 3, showing that the peak dose of the deposited energy would just melt the front surface of target material.

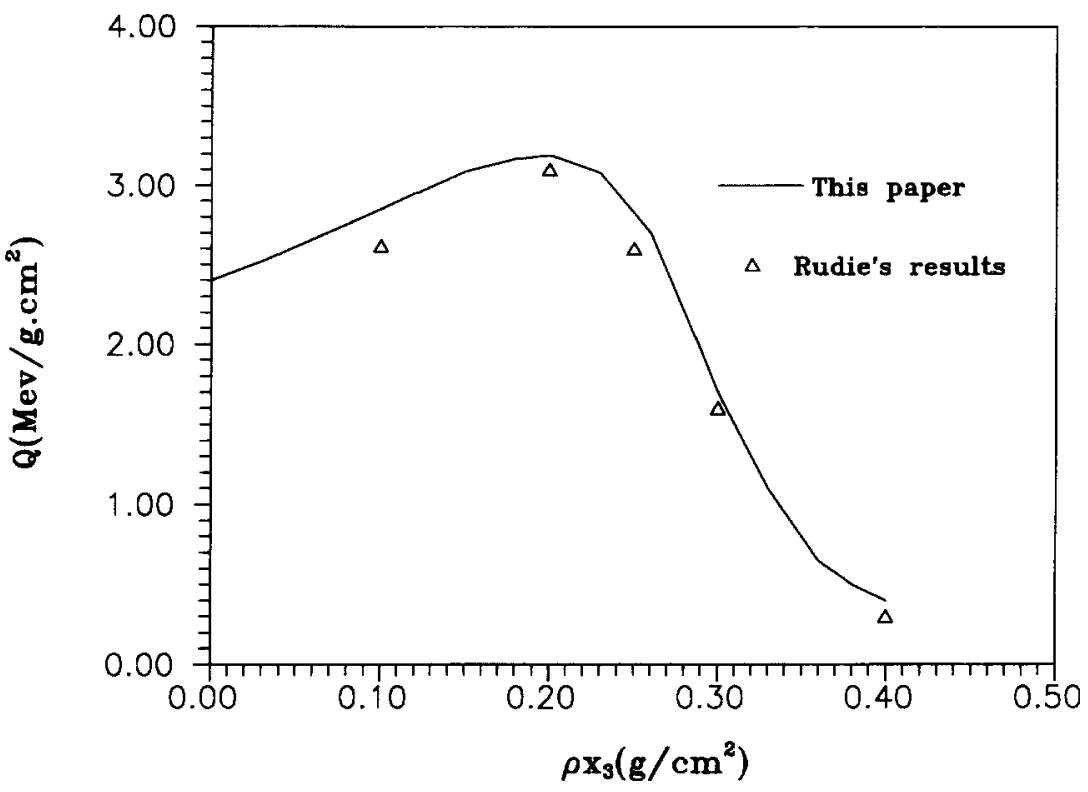

Fig. 2. Energy deposition for $1.0 \mathrm{Mev}$ electron beam in an aluminium material.

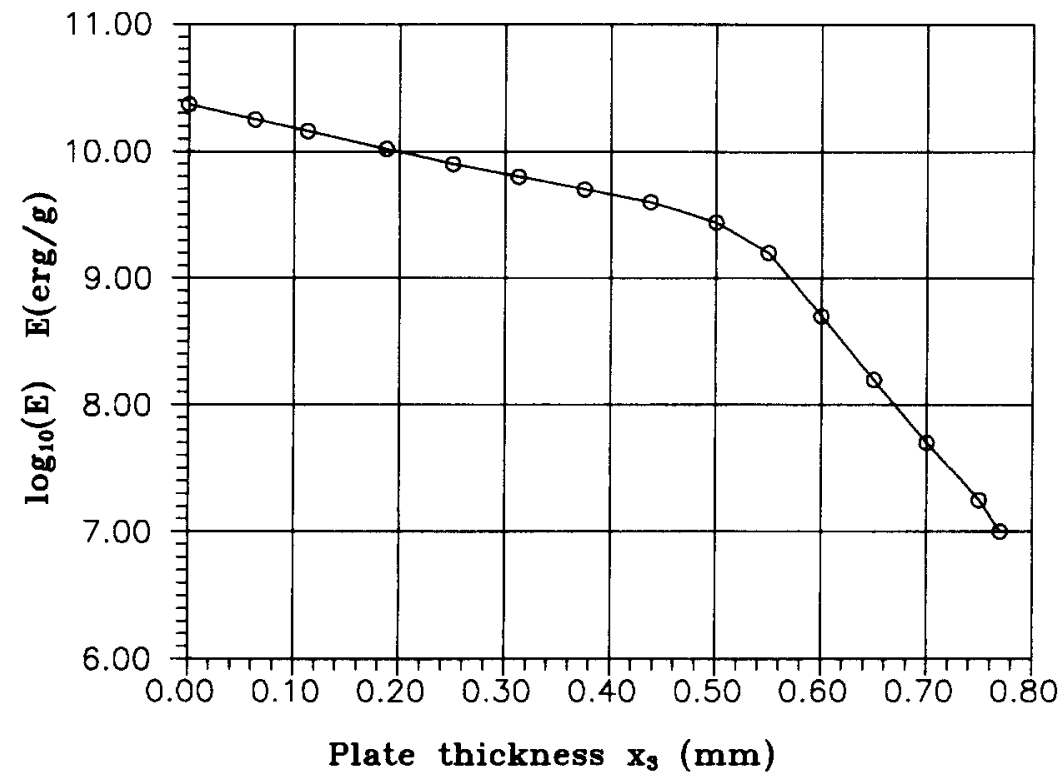

Fig. 3. Specific energy taken at the time of ending of electron beam radiation. 
This prediction is in reasonable agreement both with the observed values [32] in the fluency region from 30 to $40 \mathrm{cal} / \mathrm{cm}^{2}$, and with the mass loss near the front surface due to the pulsed beam deposition. Hence, the material target, especially in the heated surface region belongs to the intermediate region, i.e. for the pressures and temperatures near the melting transition.

The computational and experimental values for the attenuation of the $\sigma_{33}$ compressive wave peak are shown in Fig. 4. From this figure a very strong attenuation is seen nearby the incident surface. Also, this figure shows the magnitude for the calculated values higher than the measured ones. The difference is possibly due to the deviation in the calculation of beam energy deposition. As seen in Fig. 2, the calculated values are higher than the observed values for electron energy deposition in aluminium alloy. The computations for the $\sigma_{33}$ normal compressive wave profiles are shown in Fig. 5. The attenuation is clearly seen from this figure as the wave front moving across the plate. The widening of the plastic wave

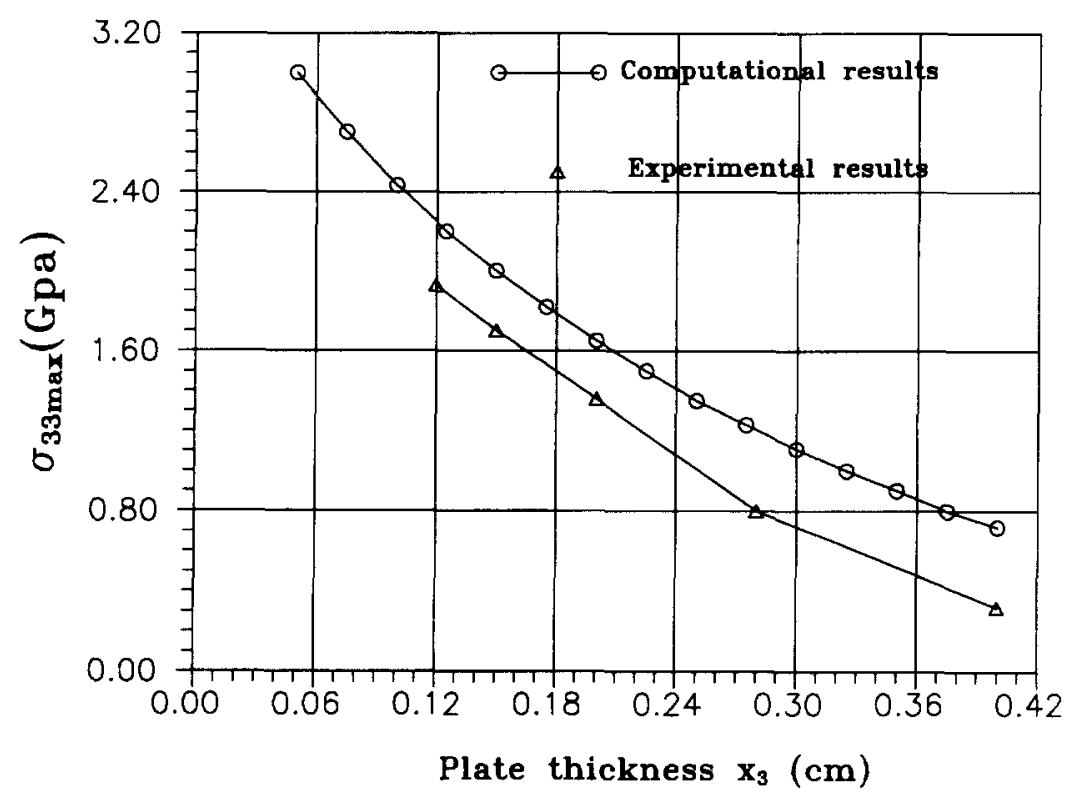

Fig. 4. Attenuation of the $\sigma_{33}$ compressive stress wave peak.

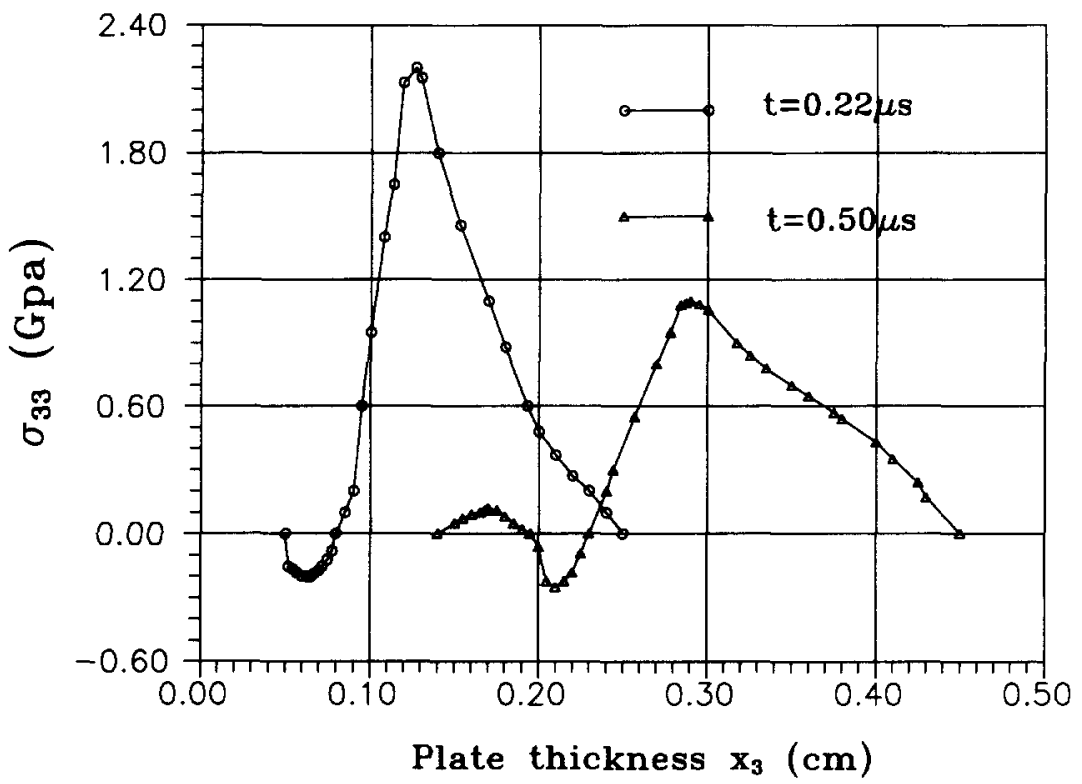

Fig. 5. Normal compressive wave profiles for the $\sigma_{33}$ component. 
front, that is, the reduction in steepness and the rounding at the top manifests the inelastic behaviour of the material.

We also give the distance-time plot of the wave profiles in Fig. 6. Regions I and II in this plot represent the hot liquid region and liquid, respectively. These liquids are blown off as a result of the thermal pressure. Regions III and IV represent the molten and solid region, respectively. As a result of heat conduction, the molten region will vanish with the propagation of the compressive stress wave. The phenomenon is regarded as the resolidification of the molten region. In the experiment, the "blown off" and the resolidification of the target materials could be observed. Their thickness of resolidified material agrees approximately with the calculated results. As seen from the above mentioned process, the incident region close to the front surface is complicated.

Figure 7 illustrates the evolution of the damage, i.e the microvoid volume increasing across the target plate, which is caused by the propagating tensile stress wave. These curves indicate that ductile spall

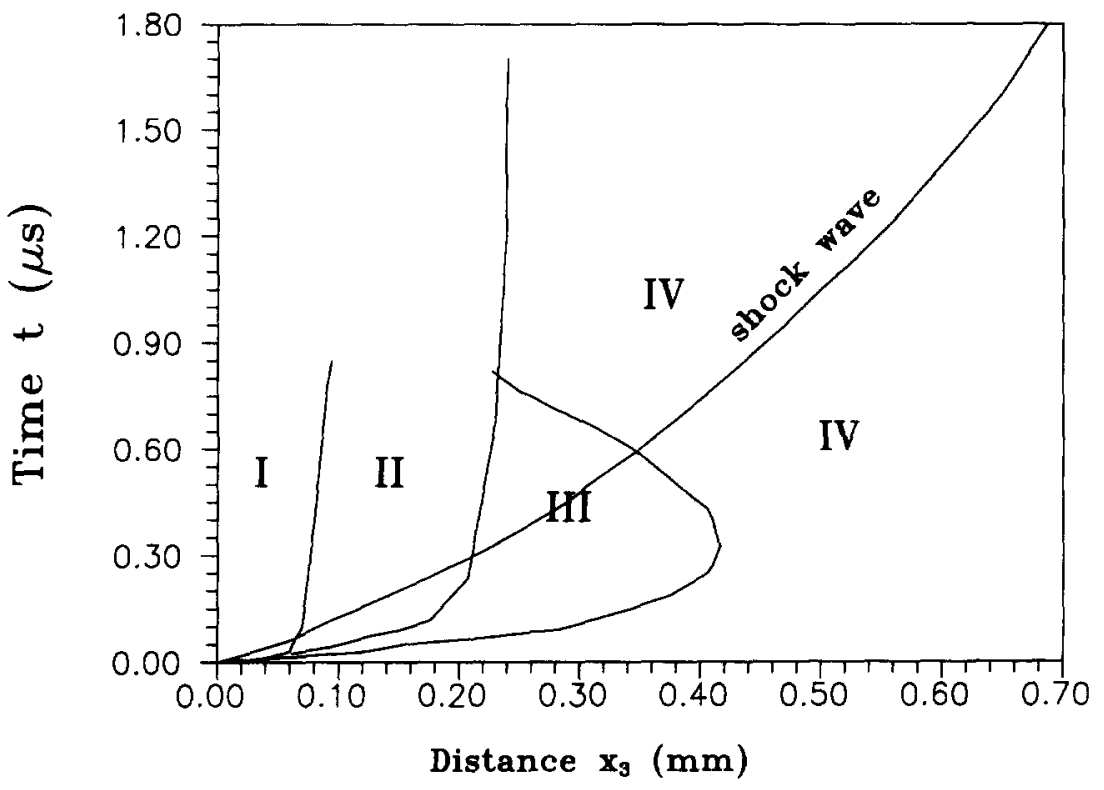

Fig. 6. Distance-time plot (1-hot liquid: II-liquid; III-melt; and IV--solid).

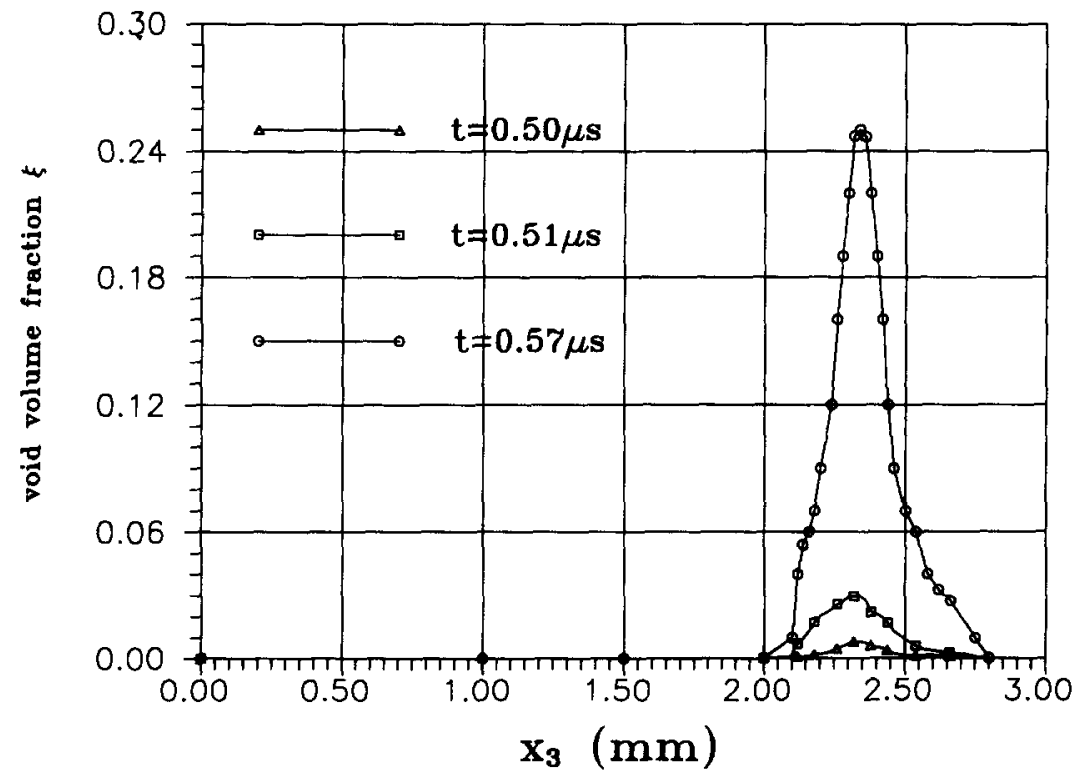

Fig. 7. Computational void volume evolution across the target plate. 
Table 2. The experimental and computational results of the thickness of spall fragements

\begin{tabular}{llllll}
\hline$h(\mathrm{~mm})$ & 1.2 & 1.5 & 2.0 & 2.8 & 4.0 \\
$h_{e}(\mathrm{~mm})$ & 0.34 & 0.35 & 0.39 & 0.43 & 0 \\
$h_{c}(\mathrm{~mm})$ & 0.41 & 0.41 & 0.42 & 0.46 & 0.48 \\
\hline
\end{tabular}

$h$ is target thickness.

$h_{e}$ is the experimental results of the thickness of spall fragments.

$h_{c}$ is the computational results of the thickness od spall fragments.

fracture will occur near the rear surface of the plate. These calculations also show that spall process lasts approximately a tenth of a microsecond from the beginning of damage development to the critical void volume fraction $\xi_{F}=0.25$. Table 2 gives the theoretical and experimental results of the thickness of spall fragments. The spall fragment becomes thicker with the increase in target thickness. This is due to the fact that the amplitude of tensile stress is much greater than the failure strength if the target is thinner. Therefore, the cumulative damage time is so short that the spall fragments become thinner. Whereas when the target is thick, the widening of the compressive stress wave increases, the tensile stress is just a little greater than the failure strength and therefore, the cumulative damage time is so much longer that the spall fragments become thicker. However, the spallation could no longer take place as the thickness of the target reaches a certain value. The theoretical and experimental studies all show that the spallation happens only once. As seen in Table 2, the spall fragment thickness calculated is a little thicker than that of the experimental data. This is probably due to the inaccurate choice for the void volume fraction $\xi=\xi_{F}$ as well as the two-dimensional effect.

\section{CONCLUSIONS}

A simple model for treating the energy deposition of electron beams on a target surface and the constitutive model with damage for describing the viscoplastic behaviour of materials are proposed. The microvoid volume fraction is taken to represent the scalar damage variable. The models have been successfully applied to examine the thermal stress wave propagation, ductile material damaging process as well as spalling for aluminium alloy.

The energy deposition of electron beams is calculated in aluminium alloy, and the temperature and pressure in the target material are shown to be within the intermediate region. The pressure in this region is governed mainly by the scaling-law from the three-phase equation of state. The agreement of the calculated and observed results for the compressive wave attenuation and spall fragments thickness indicates that the modified viscoplastic-damage constitutive relations based on the Bodner-Partom original formula seems reasonable for studying the current problem. The constitutive model suggested also signifies that the plastic volume deformation for the voided material does exist. Since the failure process involves many multi-interrelated physical mechanism, much work is left for the further investigation, including: (a) a more reasonable constitutive law, damage evolution and failure criterion used for the theoretical and numerical analysis; (b) development of 2-D FEM dynamic code for a sophisticated numerical simulation; and (c) an elaborate experimental programme for measuring the instantaneous damage evolution process, mass loss and velocity of rear surface.

Acknowledgements - The research program is supported by the NNSF of China and the Education Commission of Hunan province. These supports are fully appreciated

\section{REFERENCES}

1. Gilath, I., Eliezer, S. and Bar-Noy, T., Hemispherical shock wave decay in laser-matter interaction, Laser and Particle Beams, 1993, 11(1), 221-225.

2. Gupta, V., Measurement of interface strength by a laser spallation technique, Journal of the Mechanics and Physics of Solids, $1992,40(1), 141-180$.

3. Eliezer, S., Gilath, I. and Bar-Noy, T., Laser induced spall in metals: Experiment and simulation, Joumal of Applied Physics, 1990, 67(2), 715-724.

4. Gupta, V. and Yuan, J., Measurement of interface strength by the modified laser spallation technique, Journal of Applied Physics, 1993, 74(4), 2379-2404. 
5. Duan Z. P. and Zhou Y.C., On laser induced reverse plugging effect. Proceedings of IUTAM Symposium on Impact Dynamics (Ed. Z. M. Zheng et al.). Peking University Press, Peking, pp. 176-186 (1994).

6. Raptis, I., Glezos, N. and Hatzakis, M., Analytical evalution of the energy deposition function in electron-beam lithography in the case of a composite substrate, Journal of Vaccuum Science and Technology, B, 1993, 11(6), 2754-2757.

7. Elmer, J.W., Giedt, W.H. and Eagar, T.W., The transition from shallow to deep penetration during electron beam welding, Welding Journal, 1990, 69(5), 167s-175s.

8. Rudie J. N., Principles and Techiques of Radiation Hardening. Western Periodicals Company California, Vol 1, Chap. 2, Appendix A (1976).

9. Glezos, N., Raptis, I., Tsoukalas, D. and Hatzakis, M., Application of a new analytical techinque of electron distribution calculations to the profile simulation of a high sensitivity negative electron-beam resist, Journal of Vaccuum Science and Technology, B, 1992, 10(6), 2606-2609.

10. Oswald Jr, R.B., Mclean, F.B., Schallhom, D.R. and Oldham, T.R., Dynamic response of aluminium to pulsed energy deposition in the melt-dominated regime, Journal of Applied Physics, 1973, 44(3), 3563-3574.

11. Royce E. B., GRAY, A three-phase equation of state for metals, UCRL-51121, Lawrence Livermore Laboratory (1971).

12. Bodner S. R., Review of a unified elastic-viscoplastic theory, Unified Constitutive Equation for Creep and Plasticity. Ed. A.K. Miller, Elsevier, London, pp. 273-301 (1987).

13. Bodner S. R., A procedure for including damage in constitutive equations for elastic-viscoplastic work-hardening materials. Proceedings of the IUTAM Symposium on Physical Nonlinearities in Structural Analysis. Ed. J. Hult and J.Lemaitre, SpringerVerlag Berlin, pp. 21-28 (1981).

14. Bodner, S.R. and Partom, Y., A large deformation elastic-viscoplastic analysis of a thick-walled spherical shell, ASME Journal of Applied Mechanics, 1972, 39(3)(3), 751-757.

15. Bodner, S.R, and Partom, Y., Constitutive equations for elastic-viscoplastic strain-hardening materials, ASME Journal of Applied Mechanics, 1975, 42(2), 385-389.

16. Gurson, A.L., Continuum theory of ductile rupture by void nucleation and growth, part I-yield criteria and flow rules for porous ductile media, Journal of Engineering Materials Technology, 1977, 99(1), 2-15.

17. Subba Rao, B.N., A simple formula for the transmission and adsorption of monoenergetic electrons, Nuclear Instrumentation Mechanics, 1966, 44, 155-156.

18. Coleman, B.D. and Gurtin, M.E., Thermodynamics with internal state variable, Journal of Chemicals Physics, 1967, 47(5), 597-613.

19. LublinerJ., On the thermodynamic foundation of non-linear solid mechanics, International Journal of Non-Linear Mechanics, $1972,7(2), 237-254$.

20. Johnson, J.N., Dynamic fracture and spallation in ductile solids, Journal of Applied Physics, 1981, 52(4), 2812-2825.

21. Tvergaard, V., Influence of voids on shear band instabilities under plane strain conditions, International Journal of Fracture, 1981, 17(4), 389-407.

22. Tvergaard, V., On localization in ductile materials containing spherical voids, International Journal of Fracture, 1982, 18(4), 237-252.

23. Tvergaard, V. and Needleman, A., Analysis of the cup-cone fracture in a round tensile bar, Acta Metallicas, 1984, 32(1), 157169.

24. Chu, C.C. and Needleman, A., Void nucleation effects in biaxially stretched sheets, Journal of Engineering Materials for Technology, 1980, 102(3), 249-256.

25. Carrol, M.M. and Holt, A.C., Suggested modifications of the $P-\alpha$ model for porous materials, Journal of Applied Physics, 1972, 43(2), 759-761.

26. Seaman, L., Curran, D.R. and Shockey, D.A., Computational models for ductile and brittle fracture, Journal of Applied Physics, 1976, 47(11), 4814-4826.

27. Curran, D.R., Seaman, L. and Shockey, D.A., Dynamic failure of solids, Physics Reports, 1987, 147(5-6), 253-388.

28. Pan, J., Saje, M. and Needleman, A., Localization of deformation in rate sensitive porous solids, International Journal of Fracture, 1983, 21(4), 261-278.

29. Zhou, Y.C. and Peng, C.X., Estimation of the thermal shock wave and spall of aluminium alloy irradiated by an electron beam, Journal of High Pressure Physics (in Chinese), 1990, 4(3), 234-240.

30. Bodner, S.R., Naveh, M. and Merzer, A.M., Deformation and buckling of axisymmetric viscoplastic shells under thermomechanical loading, International Journal of Solids Structures, 1991, 27(15), 1915-1924.

31. Koplik, J. and Needleman, A., Void growth and coalescence in porous plastic solids, International Journal of Solids Structures, $1988,24(8), 835-853$.

32. Peng, C.X., Experimental studies of the thermal shock wave produced in aluminium alloy bombarded by electron beam, Explosion and Shock Waves (in Chinese), 1987, 7, 250-256. 